\section{() OPEN ACCESS}

\title{
A purified membrane protein from Akkermansia muciniphila or the pasteurised bacterium blunts colitis associated tumourigenesis by modulation of $\mathrm{CD}^{+} \mathrm{T}$ cells in mice
}

\author{
Lijuan Wang, ${ }^{1}$ Lei Tang, ${ }^{1}$ Yiming Feng, ${ }^{1}$ Suying Zhao, ${ }^{2}$ Mei Han, ${ }^{2}$ Chuan Zhang, ${ }^{3}$ \\ Gehui Yuan, ${ }^{1}$ Jun Zhu, ${ }^{1}$ Shuyuan Cao, ${ }^{1}$ Qian Wu, ${ }^{1}$ Lei Li, ${ }^{1}$ Zhan Zhang () ${ }^{1}$
}

\section{- Additional material is published online only. To view please visit the journal online (http://dx.doi.org/10.1136/ gutjnl-2019-320105). \\ ${ }^{1}$ Center for Global Health, Nanjing Medical University, Nanjing, Jiangsu, China ${ }^{2}$ Department of laboratory medicine, The Affiliated Hospital of Nanjing University of Traditional Chinese Medicine, Nanjing, China ${ }^{3}$ Department of General Surgery, Jiangsu Province People's Hospital and Nanjing Medical University First Affiliated Hospital, Nanjing, Jiangsu, China}

\section{Correspondence to} Dr Zhan Zhang, Center for Global Health, Nanjing Medical University, Nanjing 211166 , China; zhanzhang@njmu.edu.cn and Professor Lei Li, Center for Global Health, Nanjing Medical University, Nanjing, Jiangsu, China; lilei@njmu.edu.cn

LW, LT and YF contributed equally.

Received 16 October 2019 Revised 20 January 2020 Accepted 16 February 2020 Published Online First 13 March 2020

\section{ABSTRACT}

Objective Gut microbiota have been linked to inflammatory bowel disease (IBD) and colorectal cancer (CRC). Akkermansia muciniphila (A. muciniphila) is a gram-negative anaerobic bacterium that is selectively decreased in the faecal microbiota of patients with $\mathrm{IBD}$, but its causative role and molecular mechanism in blunting colitis-associated colorectal cancer (CAC) remain inconclusive. This study investigates how $A$. muciniphila engages the immune response in CAC. Design Mice were given dextran sulfate sodium to induce colitis, followed by azoxymethane to establish CAC with or without pasteurised A. muciniphila or a specific outer membrane protein (Amuc_1100) treatment. Faeces from mice and patients with IBD or CRC were collected for 16S rRNA sequencing. The effects of $A$. muciniphila or Amuc_1100 on the immune response in acute colitis and CAC were investigated. Results $A$. muciniphila was significantly reduced in patients with IBD and mice with colitis or CAC. A. muciniphila or Amuc_1100 could improve colitis, with a reduction in infiltrating macrophages and $\mathrm{CD} 8^{+}$ cytotoxic T lymphocytes (CTLS) in the colon. Their treatment also decreased CD16/32 $2^{+}$macrophages in the spleen and mesenteric lymph nodes (MLN) of colitis mice. Amuc_1100 elevated PD- $1^{+}$CTLs in the spleen. Moreover, A. muciniphila and Amuc_1100 blunted tumourigenesis by expanding CTLs in the colon and MLN. Remarkably, they activated CTLs in the MLN, as indicated by TNF- $\alpha$ induction and PD-1downregulation. Amuc_1100 could stimulate and activate CTLs from splenocytes in CT26 cell conditioned medium. Conclusions These data indicate that pasteurised $A$. muciniphila or Amuc_1100 can blunt colitis and CAC through the modulation of CTLs.

\section{INTRODUCTION}

Colorectal cancer (CRC) is the fourth leading cause of cancer-associated death worldwide. ${ }^{1}$ The initiation and progression of CRC involves complex interactions between genetics, diet, life style and environmental factors. ${ }^{2}$ Inflammatorybowel disease (IBD), including Crohn's disease and ulcerative colitis (UC), is a known risk factor for the development of CRC, namely, CAC. ${ }^{34}$ For every 10 units that in the inflammatory burden index increases in

\section{Significance of this study}

What is already known on this subject?

- The abundance of Akkermansia muciniphila is significantly decreased in patients with inflammatory bowel disease and in mice with colitis.

- A. muciniphila exerts anti-inflammatory effects on chronic colitis.

- Amuc_1100, a specific outer membrane protein from $A$. muciniphila, is stable at temperatures used for pasteurisation, can improve the gut barrier and partly recapitulates the beneficial effects of $A$. muciniphila.

What are the new findings?

- A. muciniphila abundance was decreased in mice with colitis-associated colorectal cancer (CAC).

- A. muciniphila and Amuc_1100 improved colitis by decreasing colon infiltrating macrophages and cytotoxic T lymphocytes (CTLs) in colitis mice.

- A. muciniphila and Amuc_1100 blunted CAC through the expansion and activation of CTLs, which is demonstrated by TNF- $\alpha$ induction and PD-1 downregulation.

How might it impact on clinical practice in the foreseeable future?

- Pasteurised A. muciniphila or Amuc_1100 is expected to be a new strategy to prevent and treat colitis and colorectal cancer.

patients with IBD, the risk of CRC is increased by $2.1 \% .^{5}$ Changes in the composition of the gut microbiota have been associated with an increased risk of IBD and CRC. ${ }^{67}$ Fusobacterium nucleatum can increase CRC cell proliferation and tumour development and also promote CRC chemoresistance by modulating autophagy. ${ }^{89}$ Peptostreptococcus anaerobius could promote colorectal carcinogenesis and modulate tumour immunity. ${ }^{10}$ However, in many studies, the focus has been on the putative harmful effects of the microbiota, while their beneficial effects are somewhat neglected. 


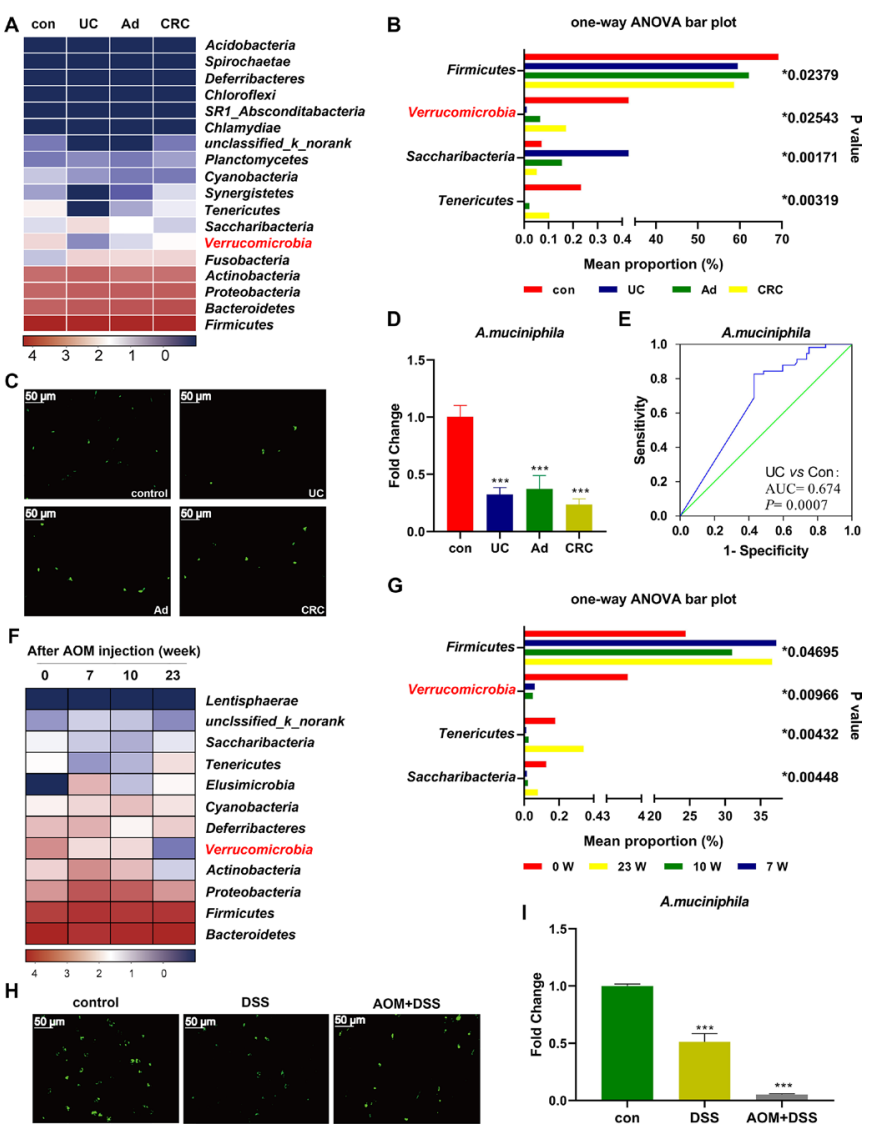

Figure 1 Decreased $A$. muciniphila was associated with colitis and CRC in human and mice. (A) Heatmap of faecal microbiota from patients with UC, Ad, CRC and healthy controls. (B) Comparison of the faecal microbiota among UC, $\mathrm{Ad}, \mathrm{CRC}$ and controls at the phylum level. (C) FISH detection of $A$. muciniphila in the colon of human patients using a FITC-conjugated $A$. muciniphila specific probe (green). Scale bars, $50 \mu \mathrm{m}$. (D) qPCR validation of the relative abundance of $A$. muciniphila in human patients. (E) ROC curves of $A$. muciniphila in predicting UC. (F) Heatmap of faecal microbiota from mice with CAC at different time points. (G) Comparison of faecal microbiota in CAC mice at the phylum level. (H) FISH detection of $A$. muciniphila in the mice with colitis and CAC (at week 23). (I) The relative abundance of $A$. muciniphila in colitis and CAC (at week 23) mice by qPCR. Data are presented as the mean \pm SEM and were analysed by ordinary one-way ANOVA with Tukey's multiple comparisons. ${ }^{* *} P<0.001$. Ad, adenoma; ANOVA, analysis of variance; $C A C$, colitis-associated colorectal cancer; $C R C$, colorectal cancer; UC, ulcerative colitis

Akkermansia muciniphila is a gram-negative anaerobic bacterium, which is the first and only representative member of the Verrucomicrobia phylum found in the human intestinal tract. ${ }^{11} 12$ Recently, it has been considered as a promising probiotics. The abundance of $A$. muciniphila is significantly reduced in metabolic disorders, such as diabetes and obesity. ${ }^{13-15}$ Supplementation with pasteurised A. muciniphila improved metabolic dysfunctions and the integrity of intestinal barrier and reduced plasma lipopolysaccharide levels in obese human volunteers. ${ }^{16} \mathrm{~A}$. muciniphila induced antigen-specific $\mathrm{T}$ cell responses to modulate host immune function during homeostasis. ${ }^{17}$ In addition, A. muciniphila restored the efficacy of programmed death-1 (PD-1) blockades by increasing the recruitment of CCR9 ${ }^{+} \mathrm{CX}-$ $\mathrm{CR} 3{ }^{+} \mathrm{CD}^{+} \mathrm{T}$ lymphocytes into mouse tumour beds. ${ }^{18}$ Our previous study showed that the abundance of $A$. muciniphila was significantly decreased in dextran sulfate sodium (DSS)-induced colitis mice. ${ }^{19}$ A. muciniphila improved clinical parameters, including spleen weight, colon inflammation index and colon histological score in chronic colitis. ${ }^{20}$ Amuc_1100 is a specific protein isolated from the outer membrane of A. muciniphila, which retains biological activity at pasteurisation temperature and exerts beneficial effects. ${ }^{21}$ However, whether A. muciniphila or Amuc_1100 can improve CAC through the modulation of immune response remain inconclusive.

The present study showed that $A$. muciniphila abundance was significantly reduced in patients with UC and mice with colitis or CAC. Oral supplementation with pasteurised A. muciniphila or Amuc_1100 attenuated DSS-induced colitis and delayed the onset of CAC. A. muciniphila/Amuc_1100 decreased colonic infiltration of macrophages and the proportion of CD16/32 macrophages in the spleen and mesenteric lymph nodes (MLN) of colitis mice. Amuc_1100 also significantly decreased colon infiltrating cytotoxic $\overline{\mathrm{T}}$ lymphocytes (CTLs), as well as the proportion of CTLs in the spleen, while it increased the PD- $1^{+}$ CTL subset. For CAC, pasteurised A. muciniphila/Amuc_1100 treatment increased CTLs in colon tumours and the MLN. Their treatment increased TNF- $\alpha^{+}$CTLs and decreased the proportion of PD- $1^{+}$CTLs in the MLN. Amuc_1100 increased the proportion of CTLs in primary splenocytes cultured in CT26 cell conditioned medium in vitro. Furthermore, these CTLs induced higher apoptotic rates than cells without Amuc_1100 treatment. Collectively, these results highlight the protective effects of Amuc_1100 in CAC, which occur through modulation of the proportion and function of CTLs. This study provides a novel evidence that $A$. muciniphila can serve as a potential therapeutic target in patients with CRC.

\section{MATERIALS AND METHODS}

\section{Culture and pasteurisation of $A$. muciniphila}

A. muciniphila MucT (ATCC BAA-835) (online supplementary figure $1 \mathrm{~A}$ ) was cultured in brain heart infusion broth containing $10 \mathrm{mg} / \mathrm{L}$ resazurin (an oxidation-reduction indicator) under strict anaerobic conditions. A representative culture stock was used to determine the $\mathrm{CFU} / \mathrm{mL}$ under anaerobic conditions by plate counting using mucin media containing 1\% agarose. This culture was diluted with anaerobic PBS containing $2.5 \%$ glycerol to a final concentration of $1.5 \times 10^{8} \mathrm{CFU} / 100 \mu \mathrm{L}$. Then, A. muciniphila was inactivated by pasteurisation for $30 \mathrm{~min}$ at $70^{\circ} \mathrm{C}$. After pasteurisation, no viable A. muciniphila could be recovered in culture.

\section{Expression and purification of Amuc_1100 in vitro}

Amuc 1100 protein was produced according to a previous study. $^{\overline{2}}$ The PCR product of a His-tagged Amuc_1100 was cloned into the pET-26b (+) vector (GenScript, Nanjing, China) to construct the expression plasmid (online supplementary figure $1 \mathrm{~B}$ and $\mathrm{C}$ ), which was validated by visualising Mlu I and Xho I digested fragments on a agarose gel (online supplementary figure 1D). The plasmid pET-26b-Amuc_1100 was transformed into E. coli BL21 (DE3), which was cultured in LB broth with kanamycin $(50 \mu \mathrm{g} / \mathrm{mL})$ at $37^{\circ} \mathrm{C}$ and $220 \mathrm{rpm}$ shaking. Isopropyl $\beta$-D-1-thiogalactopyranoside $(1 \mathrm{mM})$ was added to the LB broth to induce Amuc_1100 expression during the logarithmic growth phase. The bacteria were harvested when the OD600 value was over one and then lysed by sonification, the centrifugal supernatant was used to purify the Amuc_1100 protein with Ni-16NTA His·Bind (Novagen, Merck Millipore, Massachusetts, USA). The result of Coomassie brilliant blue staining (online supplementary 
A

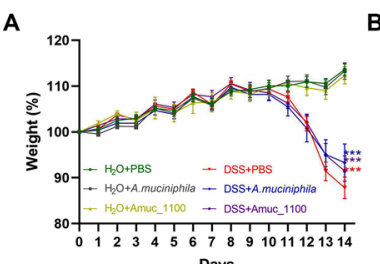

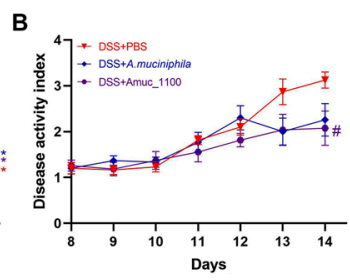
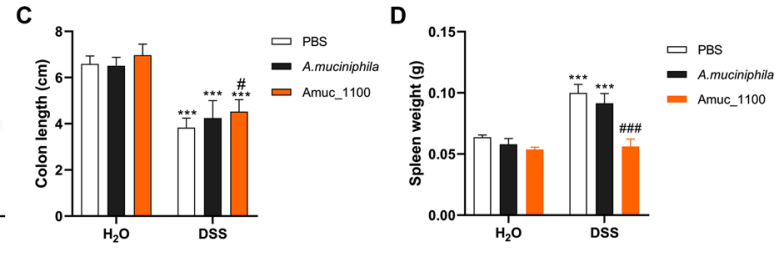

$\mathbf{E}$

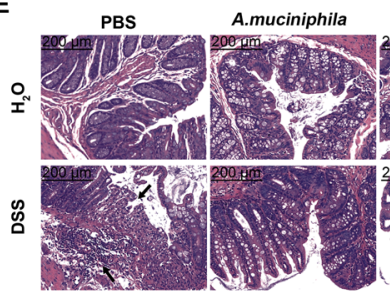

G
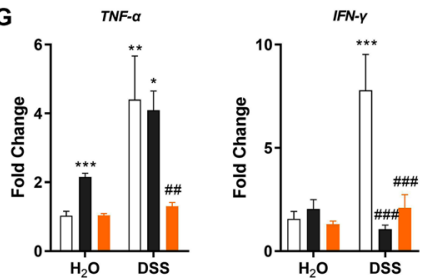

F

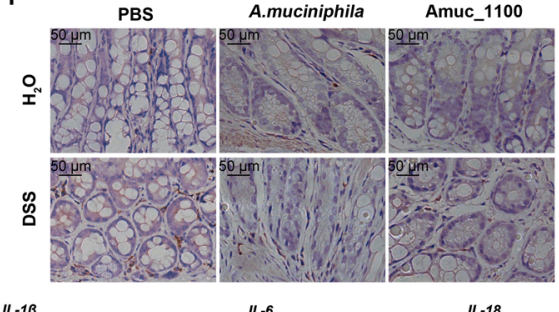

LL-1 $\beta$
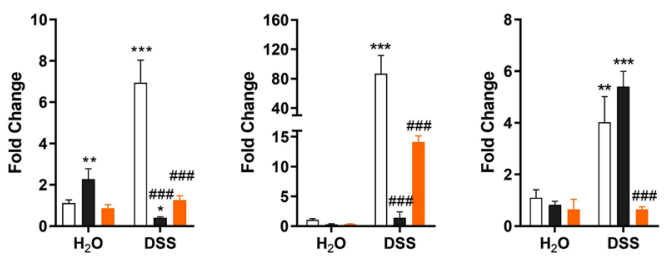
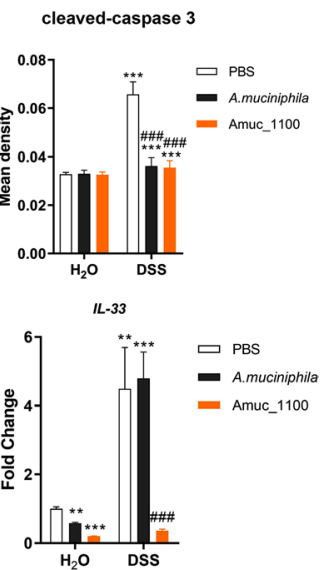

Figure 2 Supplementation with pasteurised A. muciniphila or Amuc_1100 improved colitis induced by DSS. (A) Weight changes are expressed as the mean change from the starting weight. (B) (DAI, (C) colon length and (D) spleen weight were analysed. (E) Representative histological images of colon tissues by H\&E staining. Scale bars, $200 \mu \mathrm{m}$. (F) IHC staining and quantitation of cleaved-caspase 3 in the proximal colon. Scale bars, $50 \mu \mathrm{m}$. (G) The mRNA expression of proinflammatory cytokines in colon tissue, including TNF- $\alpha$, IFN- $\gamma$, IL-1 $\beta$, IL-6, IL-18 and IL-33. Data are presented as the means \pm SEM and were analysed by ordinary one-way ANOVA with Tukey's multiple comparisons. ${ }^{*} \mathrm{P}<0.05,{ }^{*} \mathrm{p}<0.01,{ }^{* * *} \mathrm{p}<0.001$, compared with respective control; ${ }^{\#} \mathrm{p}<0.01,{ }^{\# \# \#} \mathrm{p}<0.001$, compared with DSS group. ANOVA, analysis of variance; DAl, disease activity index; DSS, dextran sulfate sodium; IHC, immunohistochemistry.

figure $1 \mathrm{E}$ ) and western blotting (online supplementary figure $1 \mathrm{~F}$ ) revealed that the Amuc_1100 protein was synthesised successfully in vitro. The concentration of Amuc_1100 was detected by an Enhanced BCA Protein Assay Kit (Beyotime Biotechnology, China). Amuc_1100 protein was stored at $-80^{\circ} \mathrm{C}$ until use.

\section{Patients}

Human stool sample from active patients with UC, adenoma (Ad) and CRC as well as healthy controls were obtained from Jiangsu Province Hospital of Chinese Medicine (Nanjing, China) with patient informed consent under an approved institutional review board protocol. The characteristics of the patients are listed in online supplementary table 1 . All of the patients with CRC were first diagnosed and then histologically confirmed. Exclusion criteria were predefined as follows: diabetes, hypertension, coronary heart disease and a history of CRC.

\section{Animal experiments}

Male C57BL/6J mice aged to $6-8$ weeks were housed in groups of five mice per cage in a 12/12 hour day/night cycle with free access to food and water under conditions of controlled humidity $(50 \% \pm 5 \%)$ and temperature $\left(22 \pm 2{ }^{\circ} \mathrm{C}\right)$.

Acute colitis was induced by $2 \%$ dextransulfate sodium (DSS) (MP Biomedicals, molecular weight $35-50 \mathrm{kDa}$ ) in the drinking water ad libitum for eight consecutive days (online supplementary figure $2 \mathrm{~A}$ ). To explore the effects of $A$. muciniphila on colitis, the mice were treated with an oral administration of pasteurised A. muciniphila $\left(1.5 \times 10^{8} \mathrm{CFU}\right)$ or the protein Amuc_1100 $(3 \mu \mathrm{g})$ in an equivalent volume of sterile PBS containing $2.5 \%$ glycerol from 2 weeks before DSS treatment to sacrifice. Body weight and disease activity index (DAI) were observed every day according our previous study. ${ }^{22}$ The average weight loss, diarrhoea and bleeding comprised the DAI (online supplementary table 2).

The mouse CAC model was established by one intraperitoneal injection (i.p.) of $10 \mathrm{mg} / \mathrm{kg}$ azoxymethane (AOM) followed by three cycles of $2 \%$ DSS treatment ( 1 week per cycle) (online supplementary figure $3 \mathrm{~A}$ ). To test the impact of $A$. muciniphila on CAC, the mice were treated with pasteurised A. muciniphila $\left(1.5 \times 10^{8} \mathrm{CFU}\right)$ or the Amuc_1100 $(3 \mu \mathrm{g})$ from 2 weeks before AOM injection until sacrifice. Body weight and DAI were observed every day. Stool samples were collected at the $7 \mathrm{th}$, 10th, 23rd weeks. The mice were euthanised at the 8 th, 12th and 23 rd weeks.

\section{S rRNA sequencing}

Microbial DNA from stool samples was extracted, then 16S rRNA was amplified at the V3 hypervariable region. Sequencing was conducted by an Illumina MiSeq (PE300) and the data were analysed by Majorbio Bio-pharm Biotechnology (www.i-sanger. com). Operational taxonomic units (OTUs) were used for alpha diversity, richness and rarefaction curve analyses with a 0.97 threshold. In addition, partial least squares discriminant analysis (PLS-DA) was performed by Bray-Curtis based on OTU level.

\section{A. muciniphila quantification}

The abundance of $A$. muciniphila in stool samples was quantified by quantitative PCR (qPCR) in accordance with our previous study. ${ }^{19}$ Localisation of $A$. muciniphila at the surface of the intestinal mucosa in colitis and CRC was performed by fluorescent in situ hybridisation (FISH) as described previously. ${ }^{8}$ The fluorescein isothiocyanate (FITC)-labelled A. muciniphila probe (FITC-CCTTGCGGTTGGCTTCAGAT) was synthesised 

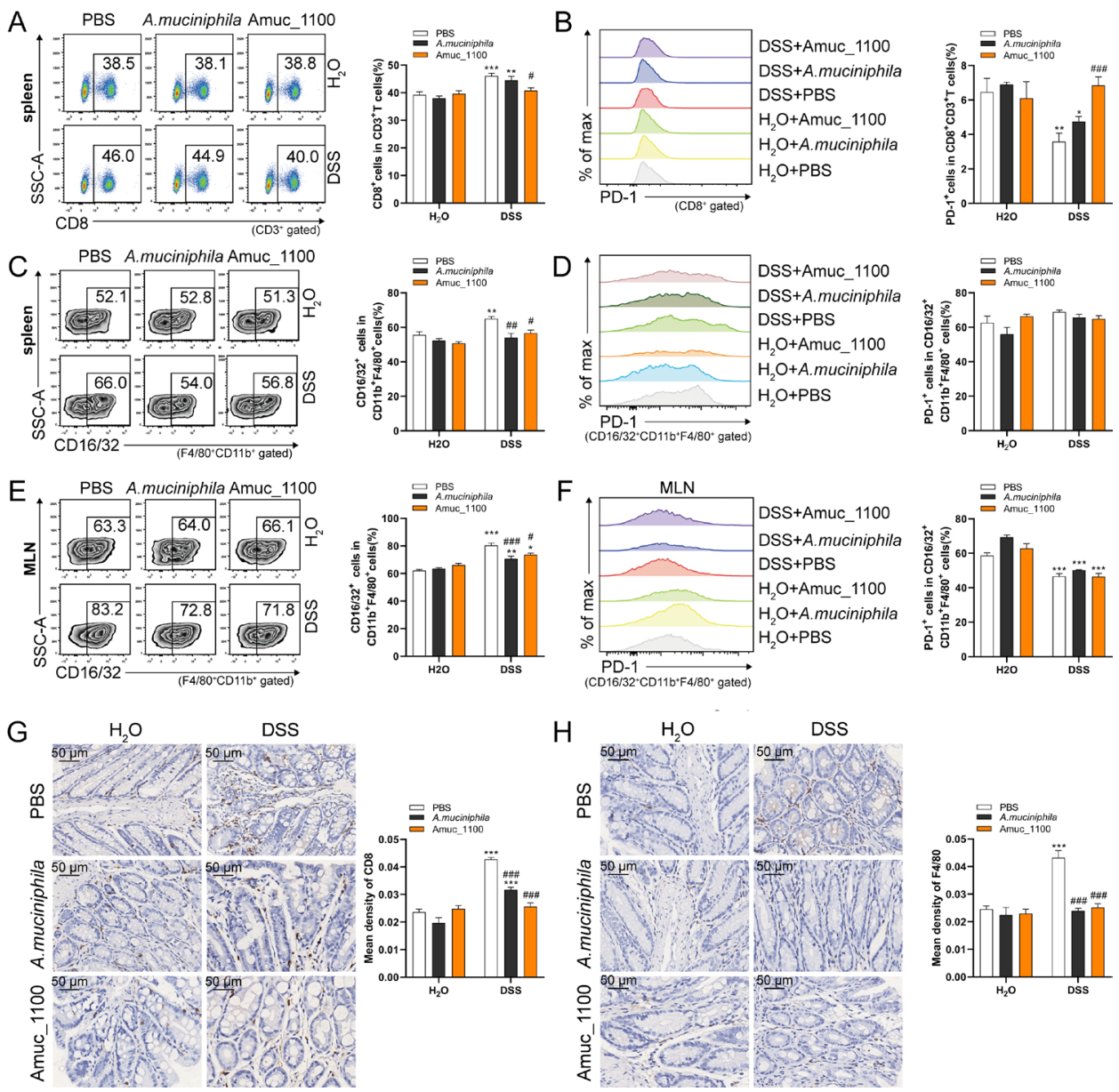

Figure 3 Supplementation with pasteurised A. muciniphila or Amuc_1100 suppressed the activation and infiltration of CD8 ${ }^{+}$CTLs and macrophages in colitis mice. Representative FCM analysis and quantification of CTLs (A) and PD1 ${ }^{+}$CTLs (B) in the spleen. Representative FCM analysis and quantification of $\mathrm{CD} 16 / 32^{+}$macrophages $\left(\mathrm{CD} 11 \mathrm{~b}^{+} \mathrm{F} 4 / 80^{+}\right)(\mathrm{C})$ and $\mathrm{PD} 1^{+} \mathrm{CD} 16 / 32^{+}$macrophages $(\mathrm{D})$ in the spleen. Scale bars, $50 \mu \mathrm{m}$. Representative FCM analysis and quantification of CD16/32 ${ }^{+}$macrophages (E) and PD1 ${ }^{+}$CD16/32 $2^{+}$macrophage (F) in the MLN. IHC staining of CD8a (G) and F4/80 $(\mathrm{H})$ in the proximal colon. Data are presented as the means \pm SEM and were analysed by ordinary one-way ANOVA Tukey's multiple comparisons. ${ }^{*} \mathrm{P}<0.05,{ }^{* *} \mathrm{p}<0.01,{ }^{* * *} \mathrm{p}<0.001$, compared with respective control; ${ }^{\#} \mathrm{p}<0.05,{ }^{\# \#} \mathrm{p}<0.01,{ }^{\# \# \#} \mathrm{p}<0.001$, compared with DSS group. ANOVA, analysis of variance; CTL, cytotoxic T lymphocytes; DSS, dextran sulfate sodium; FCM, flow cytometry; IHC, immunohistochemistry; MLN, mesenteric lymph nodes.

by GENERAY Biotechnology (Shanghai, China). Hybridised samples were imaged with a Zeiss LSM710 confocal microscope (Carl Zeiss, Germany).

\section{Quantitative real-time polymerase chain reaction (qPCR)}

Total RNA was extracted from colon tissues or CT26 cells and then the cDNA was synthesised with HiScript II Q RT SuperMix for qPCR (Vazyme Biotech). qPCR was performed using the QuantStudio 5 System (Thermo Fischer Scientific). The qPCR primers were designed and synthesised by GENERAY Biotechnology (Shanghai, China), and the primer sequences are listed in online supplementary table 3. GAPDH was used as the reference gene.

\section{Histology and immunohistochemistry (IHC)}

For histology assessment, colon tissue sections were fixed, dehydrated, embedded and sectioned $(5 \mu \mathrm{m})$ for H\&E staining. For IHC assays, the paraffin sections were deparaffinised, endogenous enzymes were inactivated and antigens were thermally repaired. The sections were then blocked and stained with CD8a, F4/80, cleaved-caspase 3, $\gamma \mathrm{H} 2 \mathrm{AX}$ or Ki67 antibodies, followed by corresponding secondary antibody and a Streptavidin Biotin
Complex kit (Boster BioEngineering, Wuhan, China). The detailed information of antibodies for IHC analysis is listed in online supplementary table 4. Stained slides were scanned by Pannoramic SCAN (3DHISTECH Kft, Budapest, Hungary). IHC was quantified by Image-Pro-Plus software and the mean density was determined based on the rate of integral optical density sum and area.

\section{Flow cytometry (FCM)}

For the analysis of CTLs, splenocytes and MLNs cells were stained with fluorescently labelled antibodies (online supplementary table 4) including Fixable Viability Dye eFlour 780, PerCP-eFluor 710 anti-mouse CD3, FITC rat anti-mouse CD8a, PE rat anti-mouse TNF, BD Horizon BV421 hamster anti-Mouse CD279. To remove non-specific binding of fluorescently labelled antibodies to $\mathrm{Fc}$ receptors, the $\mathrm{Fc}$ receptors were blocked with purified anti-mouse CD16/32. For intracellular staining of TNF- $\alpha$, the cells were treated with the Leucocyte Activation Cocktail for 12 hours. The cells were then fixed, permeabilised and washed using the Foxp3/Transcription Factor Staining Buffer Set Kit (\#85-00-5523-00, eBioscience). 
For the analysis of the macrophages, splenocytes and MLNs cells were stained with fluorescently labelled antibodies, including FITC rat anti-mouse CD11b, PE rat anti-mouse F4/80, PerCP-Cy5.5 anti-mouse CD16/32, anti-mouse CD279 (PD-1) and FcR block. Gates and quadrants were based on fluorescence minus one (FMO) control. The samples were detected with BD FACSVerse (BD Biosciences). The data were analysed by FlowJo_V10.

\section{Coculture of primary mouse CTLs with CT26 cells}

CT 26 cells were purchased from the Cell Bank of the Chinese Academy of Sciences (Shanghai, China) and cultured in RPMI1640 medium containing $10 \%$ fetal bovine serum (FBS) at $37^{\circ} \mathrm{C}$ in a $5 \% \mathrm{CO}_{2}$ humidified atmosphere. Splenocytes were isolated and cultured in fresh RPMI-1640 medium containing 20\% FBS and CT26 conditioned medium (v/v, 1/1) with or without $10 \mu \mathrm{g} / \mathrm{mL}$ Amuc_1100 for 24 hours. Then, the cultured splenocytes were used to sort CTLs by FCM. These suspended CTLs and adherent CT26 cells were cocultured at 1:1 for 48 hours. The apoptotic rate of CT26 cells was examined with the FITC Annexin V Apoptosis Detection Kit I (\#556547, BD Biosciences) according to the manufacturer's instructions.

\section{Statistical analysis}

The receiver operating characteristic curve (ROC) analysis was performed by SPSS 22.0 software (Chicago, Illinois, USA). A two-tailed Student's t-test was performed to compare the cell apoptotic rate and related gene expression in CT26 cells. All human and animal data were analysed by ordinary one-way analysis of variances with Tukey's multiple comparisons. All plots are shown as the means \pm SEM. $\mathrm{P}<0.05$ was considered as statistically significant.

\section{RESULTS}

\section{The abundance of $A$. muciniphila was decreased in human} and mice with colitis or CRC

$16 \mathrm{~S}$ rRNA sequencing indicated that the community composition could be clearly separated by PLS-DA and rarefaction curves between UC and healthy controls (online supplementary figure 4A-B). The diversity (Shannon index) and richness (Chao, ACE and Sobs indices) of the gut microbiota were significantly reduced in patients with UC in comparison with controls (online supplementary figure 4C-F). The sequencing data revealed a decreased abundance of $A$. muciniphila in patients with UC, Ad and CRC (figure 1A-B), which were verified by FISH (figure 1C) and qPCR data (figure 1D). ROC analysis revealed the predicted value of A. muciniphila in UC $(\mathrm{AUC}=0.674, \mathrm{p}=0.0007$ ) (figure $1 \mathrm{E}$ ). In agreement with the human data, the diversity and richness of the microbiota in colitis mice were significantly decreased (online supplementary figure 2C-F). Unexpectedly, supplementation with Amuc_1100 increased the abundance of A. muciniphila in colitis mice (online supplementary figure $2 \mathrm{G}-\mathrm{I}$ ). There was a complete separation between the gut microbiota from CAC mice at different time points (online supplementary figure $5 \mathrm{~A}-\mathrm{B}$ ). The diversity and richness of the gut microbiota in CAC mice were significantly reduced in the early stage (online supplementary figure $5 \mathrm{C}-\mathrm{F})$. During the process of CAC, A. muciniphila decreased in a time-dependent manner (figure $1 \mathrm{~F}-\mathrm{G}$ ). FISH and qPCR data further confirmed that $A$. muciniphila abundance was decreased in colitis and CAC mice (figure $1 \mathrm{H}-\mathrm{I}$ ).
Pasteurised $A$. muciniphila or Amuc_1100 improved colitis in mice

Oral supplementation of pasteurised A. muciniphila or Amuc_1100 failed to recover the loss of body weight in colitis mice (figure 2A). However, Amuc_1100 significantly alleviated the DAI (figure 2B). Both pasteurised A. muciniphila and Amuc_1100 significantly relieved colon shortening (figure 2C) and splenomegaly (figure 2D). They also attenuated histological injuries in the proximal colon, such as the loss of histological structure, disruption of the epithelial barrier and pronounced inflammatory cell infiltration (figure $2 \mathrm{E}$ ). IHC analysis demonstrated that pasteurised A. muciniphila or Amuc_1100 decreased the number of cleaved-caspase three positive cells (figure $2 \mathrm{~F}$ ). qPCR analyses of colon from colitis mice after their treatment revealed significant downregulation of TNF- $\alpha$, IFN- $\gamma$, IL- $1 \beta$, IL-6, IL-18 and IL-33 (figure 2G). Collectively, these results demonstrated that treatment with pasteurised A. muciniphila or Amuc_1100 could ameliorate DSS-induced acute colitis.

\section{Reduced infiltration of CTLs and macrophages in the spleen and MLN by supplementation of Amuc_1100 or pasteurised $A$. muciniphila}

To investigate the effects of A. muciniphila or Amuc_1100 on immune response, the proportion of CTLs and macrophages as well as their expressions of PD-1 and TNF- $\alpha$ were detected by FCM (online supplementary figure 6). The proportion of CTLs was significantly increased in the spleens of colitis mice (figure 3A). Oral supplementation of Amuc 1100 significantly decreased the proportion of CTLs (figure $3 \mathrm{~A}$ ) and rescued the reduced percentage of $\mathrm{PD}-1^{+}$CTLs in the spleen (figure $3 \mathrm{~B}$ ). However, A. muciniphila and Amuc_1100 could not restore the reduction in $\mathrm{CD}^{+} \mathrm{T}$ cells (online supplementary figure $7 \mathrm{~A}$ ). They inhibited the elevation in proinflammatory CD16/32 macrophages $\left(\mathrm{CD} 11 \mathrm{~b}^{+} \mathrm{F} 4 / 80^{+}\right)$in the spleen (figure $3 \mathrm{C}$ ).

The proportion of CTLs (online supplementary figure $7 \mathrm{~B}$ ) and PD $-1^{+}$CTLs (online supplementary figure 7C) did not change in the MLN with or without pasteurised A. muciniphila or Amuc 1100 treatment. However, their treatment suppressed the increase in CD16/32 $2^{+}$macrophages (figure 3E), but they did not impact the expression of PD-1 on CD16/32 ${ }^{+}$macrophages in the spleen (figure 3D) or MLN (figure 3F). Furthermore, they alleviated the colonic infiltration of CTLs (figure $3 \mathrm{G}$ ) and macrophages (figure $3 \mathrm{H}$ ).

\section{Supplementation with pasteurised $A$. muciniphila or Amuc_1100 improved clinical symptoms of CAC}

Neither pasteurised A. muciniphila nor Amuc_1100 administration could offset the reduction in body weight observed in CAC mice (figure 4A). However, they significantly decreased the DAI, especially at week 23 (figure 4B). Pasteurised A. muciniphila or Amuc_1100 did not change the colon length (online supplementary figure $3 \mathrm{~B}$ ), while their treatment could mitigate splenomegaly in CAC mice (figure 4C). In addition, their treatment delayed the tumour formation (figure $4 D, G$ and online supplementary figure $3 \mathrm{C}$ ) and decreased the tumour numbers and area by the 12th week (figure 4E-F). Their treatment also decreased the expression of $\gamma \mathrm{H} 2 \mathrm{AX}$ (figure $4 \mathrm{H}$ ) and cleaved caspase 3 (figure 4I) in colon epithelial cells, which indicated that they could attenuate double-stranded DNA breaks and subsequent apoptosis in CAC mice. Furthermore, the expression of Ki67 was reduced on pasteurised A. muciniphila and Amuc_1100 treatment (figure $4 \mathrm{~J}$ ), suggesting that they may also inhibit cell proliferation. Collectively, these data demonstrated that oral 
A

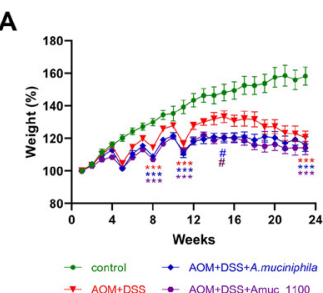

B

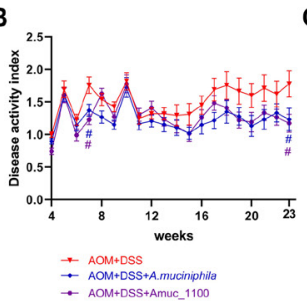

C

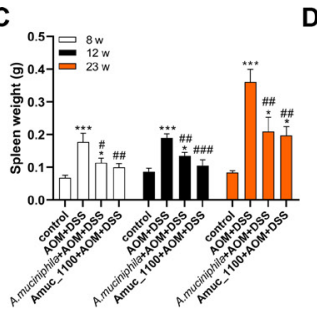

$\mathrm{H}$
D

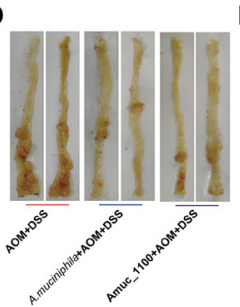

E

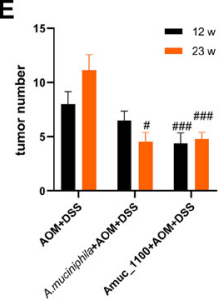

$\mathbf{F}$

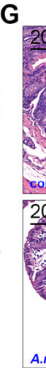

I
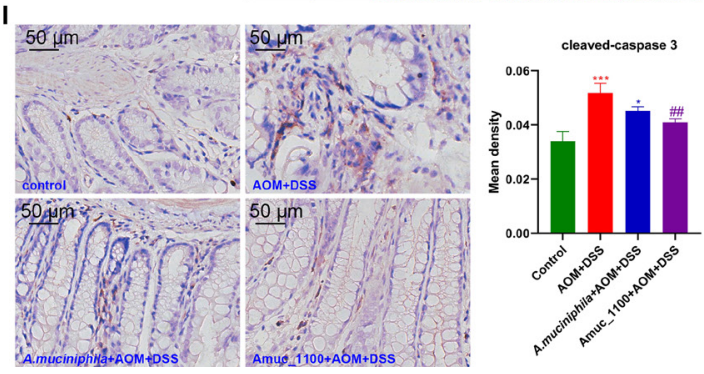

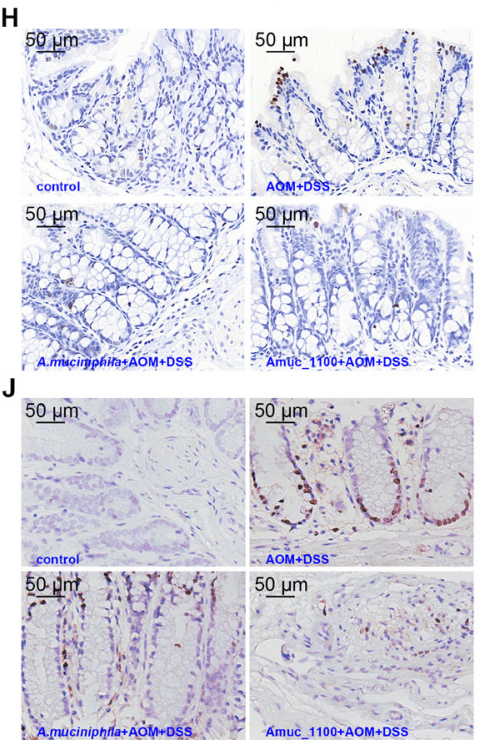

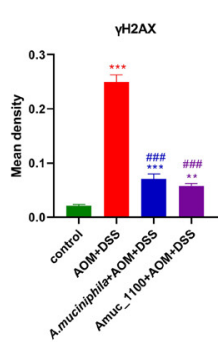

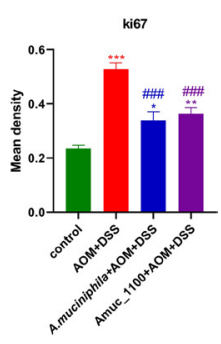

Figure 4 Supplementation with pasteurised A. muciniphila or Amuc_1100 blunted tumourigenesis induced by azoxymethane (AOM)/DSS. (A) Weight changes are expressed as the mean change from the starting weight. (B) DAl, (C) spleen weight, (D) tumour development, number (E) and tumour area (F) were analysed. (G) Representative histological images of colon tissues by H\&E staining. Scale bars, $200 \mu \mathrm{m}$. IHC staining and quantitation of $\gamma \mathrm{H} 2 \mathrm{AX}(8$ weeks, H), cleaved-caspase 3 (23 weeks, I) and Ki67 (23 weeks, J) in the proximal colon. Scale bars, $50 \mu \mathrm{m}$. Data are presented as the mean \pm SEM and were analysed by ordinary one-way ANOVA with Tukey's multiple comparisons. ${ }^{*} P<0.05,{ }^{* *} p<0.01,{ }^{* * *} p<0.001$, compared with respective control; ${ }^{\#}<<0.05,{ }^{\#} \mathrm{p}<0.01,{ }^{\# \#} \mathrm{p}<0.001$, compared with AOM+DSS group. ANOVA, analysis of variance; DAI, disease activity index; DSS, dextran sulfate sodium; IHC, immunohistochemistry.

administration of pasteurised A. muciniphila or Amuc_1100 could prevent AOM/DSS-induced tumourigenesis by attenuating DNA damage, cell apoptosis and abnormal proliferation.

\section{Pasteurised A. muciniphila or Amuc_1100 modulated immune response in CAC mice}

Studies have shown that CTLs undergo aggressive proliferation, cloning and dynamic differentiation in the human tumour microenvironment. ${ }^{23}$ The proportion of CTLs in the MLN increased significantly only after the $23 \mathrm{rd}$ week (figure $5 \mathrm{~B}$ and online supplementary figure $8 \mathrm{~B}$ ), while the proportion of PD- $1^{+}$CTLs was significantly increased by the 12 th week (figure 5A,C). Pasteurised A. muciniphila and Amuc_1100 significantly enhanced the increase in CTLs in the MLN of CAC mice (figure 5A-B), while they reduced the percentage of PD- $1^{+}$ CTLs starting in the 8th week (figure 5C). The TNF- $\alpha^{+}$CTLs cell subset percentage was significantly higher after their treatment for 8 and 12 weeks (figure 5A,D and online supplementary figure $8 \mathrm{~A}$ ). In the spleen, pasteurised A. muciniphila and Amuc_1100 significantly reduced the proportion of PD- $1^{+}$CTLs by the 8 th week (online supplementary figure 9). Furthermore, their treatment suppressed the colonic infiltration of CTLs in CAC mice (figure 5E). These data suggested that pasteurised $A$. muciniphila and Amuc_1100 increased the proportion of CTLs and enhanced their cytotoxic effect in CAC mice.

Pasteurised A. muciniphila and Amuc_1100 suppressed the increase percentage of macrophages in the spleen of CAC mice since by the 8th week (figure 6A,B), while they had no effect on the proportion of macrophages (online supplementary figure $10 \mathrm{~A}$ ) or CD16/32 $2^{+}$macrophage in the MLN (online supplementary figure 10B). However, their treatment significantly inhibited the expression of PD-1 on CD16/32 $2^{+}$macrophage in the MLN and spleen (figure 4B and online supplementary figure 10C) and alleviated the colonic infiltration of macrophages (figure 6C).

\section{Amuc_1100 induced activation of CTLs cultured in CT26 cell conditioned medium}

To explore the underlying antitumour mechanism of Amuc_1100, CTLs were isolated from the spleen of normal mice and cocultured with CT26 cells in vitro (figure 7A). Amuc_1100 significantly increased the proportion of CTLs in primary splenocytes cultured in CT26 cell conditioned medium (figure 7B). The purified CTLs pretreated with Amuc_1100 significantly increased the apoptosis of CT26 cells in the conditioned medium (figure 7C). qPCR of cocultured CT26 cells revealed a downregulation of Bcl-2 (figure 7D) and upregulation of caspase 3 (figure 7E). Intratumoural expression of cleaved-caspase 3 (figure 7F) was increased in CAC mice treated with pasteurised A. muciniphila or Amuc_1100.

\section{DISCUSSION}

The maintenance of intestinal homeostasis relies on coordinated interactions between the microbiota, the intestinal epithelium 

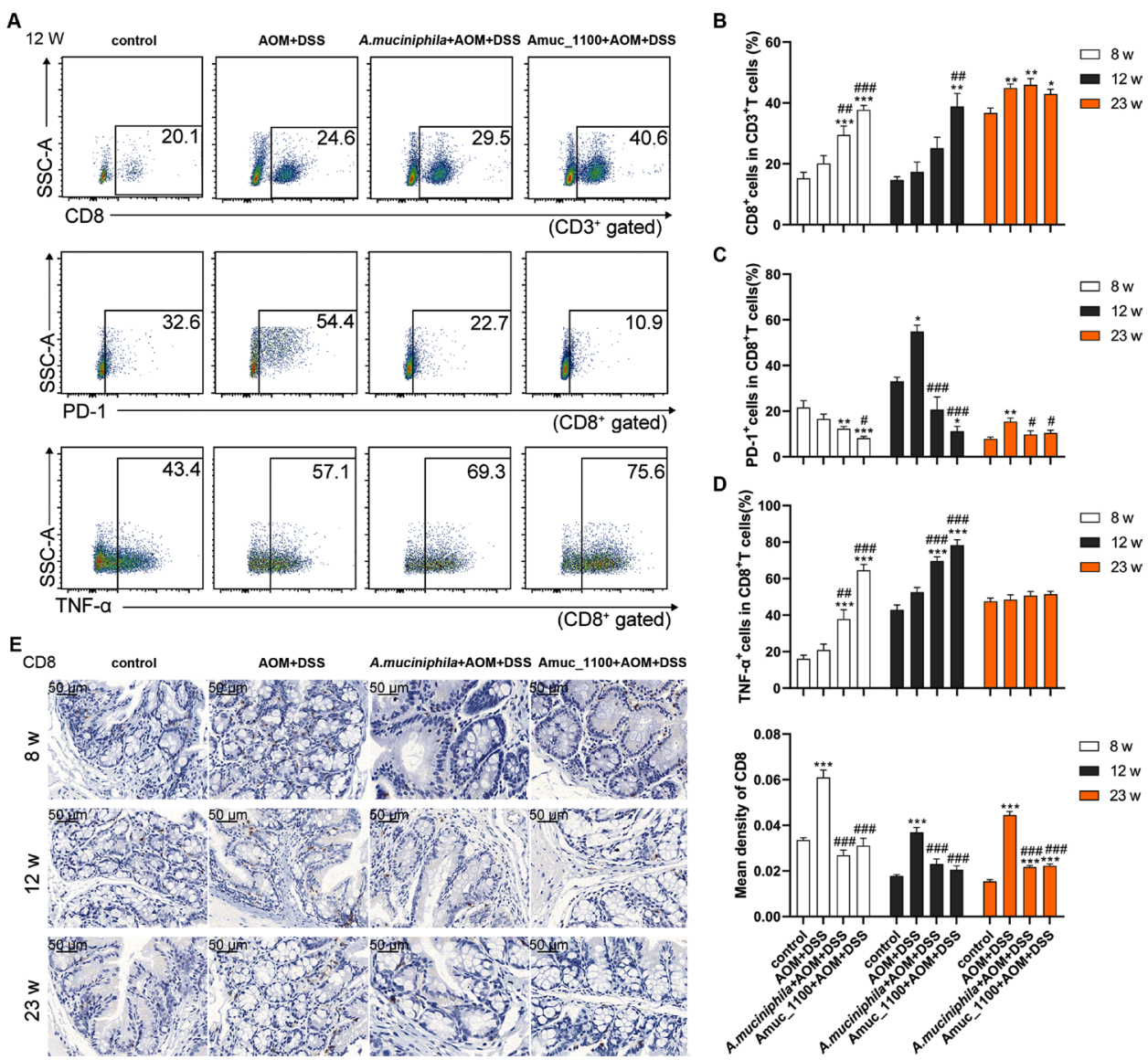

Figure 5 Pasteurised A. muciniphila or Amuc_1100 enhanced CTLs activation in CAC mice. FCM analysis of CTLs, PD-1 $1^{+}$CTLs and TNF- $\alpha^{+}$CTLs in the MLN in the 12th week (A). Quantification of CTLs (B), PD-1+ CTLs (C) and TNF- $\alpha$ CTLs (D) in the 8th, 12th and 23rd week, respectively. (E) IHC staining of CD8a in the colon. Scale bars, $50 \mu \mathrm{m}$. Data are presented as the means \pm SEM and were analysed by ordinary one-way ANOVA with Tukey's multiple comparisons. ${ }^{*} \mathrm{P}<0.05,{ }^{* *} \mathrm{p}<0.01,{ }^{* * *} \mathrm{p}<0.001$, compared with control; ${ }^{\#} \mathrm{p}<0.05,{ }^{\# \#} \mathrm{p}<0.01$, ${ }^{\# \#} \mathrm{p}<0.001$, compared with AOM+DSS group. ANOVA, analysis of variance; AOM, azoxymethane; CAC, colitis-associated colorectal cancer; CTL, cytotoxic T lymphocyte; DSS, dextran sulfate sodium; FCM, flow cytometry; IHC, immunohistochemistry.

and the host immune system. ${ }^{24}$ In this study, the microbiota composition in faeces from patients with UC was distinct from healthy individuals. However, a dramatic shift in the microbial community structure was not observed in patients with Ad or CAC because of interindividual variation and small sample size. Richness and diversity were significantly reduced in colitis and CAC mice. Consistent with previous study, ${ }^{12}$ the abundance of A. muciniphila in patients with UC and DSS-induced colitis mice was decreased. The role of A. muciniphila in CRC still remains inconclusive. Nevertheless, A. muciniphila has previously been linked to an increased burden of CRC in human and mice, ${ }^{25-27}$ and it was decreased during the progression of CAC in mice and in patients with CRC in this study. Thus, the aims of this study were to explore whether and how A. muciniphila restrain colorectal tumourigenesis. Recent studies showed that both pasteurised A. muciniphila and Amuc_1100 (stable after pasteurisation) could partly recapitulate the beneficial effects of the live bacterium. ${ }^{1621}$ Thus, they were used to validate the role of $A$. muciniphila in the development of acute colitis and CAC.

The A. muciniphila type strain ATCC BAA-835 and a murine strain (designated 139) exerted anti-inflammatory effects on chronic colitis. ${ }^{20}$ Previous studies also revealed the protective effects of A. muciniphila-derived extracellular vesicles in DSSinduced colitis. ${ }^{28}$ In this study, oral administration of pasteurised A. muciniphila or Amuc_1100 significantly improved acute colitis, including DAI, splenomegaly, colon shortening and histological injury. A. muciniphila is known to be important in improving the host metabolic functions in obese and diabetic people. ${ }^{29}$ Thus, it seemed reasonable that A. muciniphila and Amuc 1100 failed to restore the body weight loss observed in colitis or CAC mice. Their treatment also inhibited colonic expression of proinflammatory cytokines, such as IFN- $\gamma$, TNF- $\alpha$ and IL-1 $\beta$. However, the BAA-835 strain previously failed to promote short-term intestinal inflammation in gnotobiotic IL-10-deficient mice. ${ }^{30}$ Oral supplementation of pasteurised A. muciniphila or Amuc_1100 could not shape the microbial community in colitis mice, but they did enhance the abundance of A. muciniphila. It seems that the protective effects are dependent on the microbiota. CTLs are emerging as major mediators of human IBD, which is also prognostically linked to CTL profiling. ${ }^{31}$ The main contribution to colitis development was mediated by cytokine producing, proliferative, CTLs in the intraepithelial compartment of mice. ${ }^{32}$ Amuc_1100 significantly decreased the proportion of CTLs in the spleen and the colonic infiltration of CTLs. Moreover, Amuc_1100 inhibited the activation of CTLs through the upregulation of PD-1. Taken together, Amuc 1100 could improve DSS-induced colitis through the modulation of the population and activation of CTLs.

Beyond the effect on colitis, this study uncovered that Amuc_1100 provoked an antitumour immune response in CAC. Oral supplementation of pasteurised A. muciniphila or Amuc_1100 delayed tumour formation and reduced tumour 
A

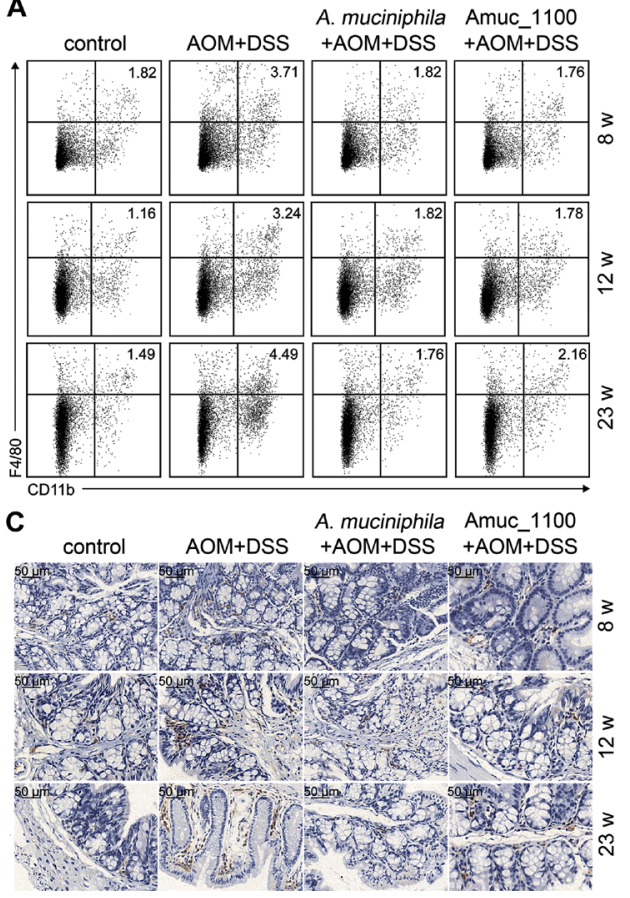

B
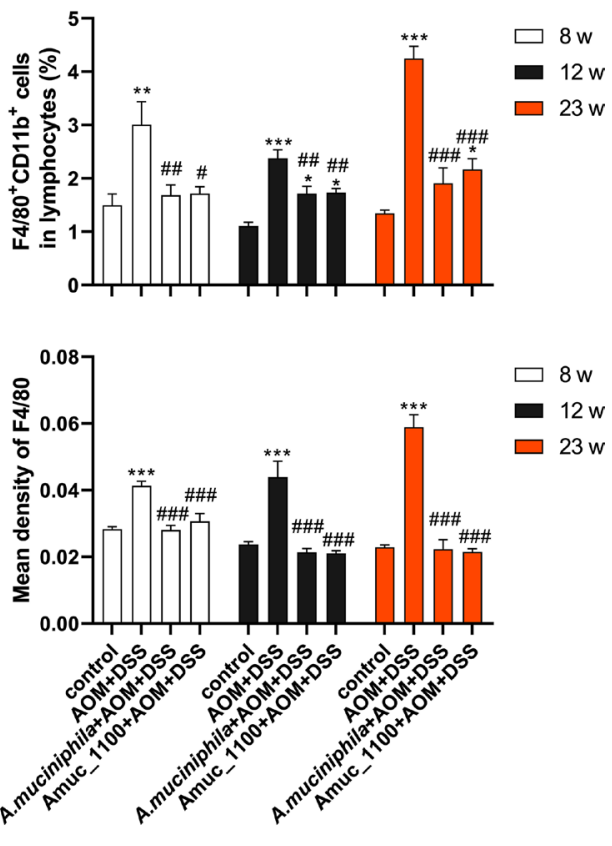

Figure 6 The reduced proportion and infiltration of macrophages in CAC mice treated with pasteurised A. muciniphila or Amuc_1100. (A) FCM analysis and (B) quantification of macrophage $\left(C D 11 b^{+} F 4 / 80^{+}\right)$in lymphocytes of the spleen in the 8th, 12th and 23rd week. (C) IHC of F4/80 in the colon. Scale bars, $50 \mu \mathrm{m}$. Data are presented as the mean \pm SEM and were analysed by ordinary one-way ANOVA with Tukey's multiple comparisons. ${ }^{*} \mathrm{P}<0.05,{ }^{* *} \mathrm{p}<0.01,{ }^{* * *} \mathrm{p}<0.001$, compared with control; ${ }^{\#} \mathrm{p}<0.05,{ }^{\# \#} \mathrm{p}<0.01,{ }^{\# \#} \mathrm{p}<0.0001$, compared with AOM+DSS group. ANOVA, analysis of variance; AOM, azoxymethane; CAC, colitis-associated colorectal cancer; DSS, dextran sulfate sodium; FCM, flow cytometry; IHC, immunohistochemistry.

number and size. As a known genotoxic stress, AOM can trigger DNA damage and subsequent cell apoptosis and abnormal proliferation. ${ }^{33}$ This study revealed that pasteurised A. muciniphila and Amuc_1100 inhibited the expression of $\gamma \mathrm{H} 2 \mathrm{AX}$ and Ki67 in colon tissue, while they triggered the apoptosis of tumour cells in vitro and in vivo. CTLs are considered the principal cellular mediators of antitumour immunity and a prognosis marker for CRC. $^{34-36}$ Pasteurised A. muciniphila and Amuc_1100 significantly increased CTL percentage in the MLN and colon. Interestingly, they also upregulated TNF- $\alpha$ in CTLs, which aggravated tumour cells apoptosis. ${ }^{37} 38$ The different microenvironment of colitis and CAC may partially explain this discrepancy. A. muciniphila and Amuc_1100 might promote the recruitment of CTLs though increasing the secretion of specific chemokines in tumour cells, where they were activated due to MHCI upregulation. ${ }^{39}$ However, the underlying mechanisms need further investigation.

Immune checkpoint inhibitors targeting the PD-1/programmed death-ligand 1 (PD-L1) axis are widely used. ${ }^{40} 41$ The composition of the commensal microbiota in patients is associated with the therapeutic efficacy of anti-PD-1 monoclonal antibody. ${ }^{42}$ Oral gavages with A. muciniphila increased the efficacy of a PD-1 blockade with respect to tumour growth. ${ }^{18}$ The expression of PD- 1 was upregulated on mucosal CD4, CD $8 \alpha \beta$ and TCR $\alpha \beta$ cells of the colon and the ileum, which correlated with CAC progression. ${ }^{43}$ Although the majority of CRCs with high CTLs infiltration have favourable outcomes, those with concurrently high PD-L1 gene expression have poor clinical outcomes. ${ }^{44}$ In the present study, the proportion of PD- $1^{+}$CTLs was significantly reduced in CAC mice treated with pasteurised A. muciniphila or Amuc_1100. T cells expressing lower levels of PD-1 produced higher levels of IFN- $\gamma$ and TNF- $\alpha$ in comparison to those with high PD-1 expression, which might in turn trigger cell apoptosis and induce PD-L1 expression in the tumour microenvironment, thereby shaping T-cell PD-1 status. ${ }^{45}$

Macrophages are the gatekeepers of intestinal immune homeostasis, M1 macrophages elicit rapid proinflammatory responses to infection and tissue damage. ${ }^{46}{ }^{47}$ In the present study, colitis was blunted by A. muciniphila or Amuc_1100 through a reduction in the proportion of macrophage, especially CD $16 / 32^{+}$macrophages (M1). Previous studies revealed that CTLs could promote the recruitment and activation of macrophages during inflammation. ${ }^{48}$ Thus, A. muciniphila or Amuc_1100 may inhibit the recruitment and activation of macrophages by the reduction of CTLs. Conversely, they could also inhibit the activation of macrophages by increased CTLs. Clinical and experimental evidence has shown that tumourassociated macrophages (TAMs) promote cancer initiation and progression. The proportion of macrophage in the spleen as well as the colonic infiltration of macrophages were significantly increased in CAC mice. A. muciniphila or Amuc_1100 treatment decreased these macrophage populations without disrupting their expression of CD16/32 or PD-1, indicating that their antitumour capabilities were independent of macrophage activation. A recent study reported that TAMs could promote CTL tumour infiltration through the production of CXCL9. ${ }^{49}$ Thus, A. muciniphila or Amuc_1100 may also inhibit macrophage functions, such as chemokines secretion, and enhance the tumour infiltration of CTLs. However, the interactions of macrophages and CTLs on A. muciniphila or Amuc_1100 treatment remain unclear.

This work reveals a key role for A. muciniphila as a critical regulator of intestinal homeostasis. Pasteurised A. muciniphila or Amuc_1100 could reshape the immune landscape to blunt 
A
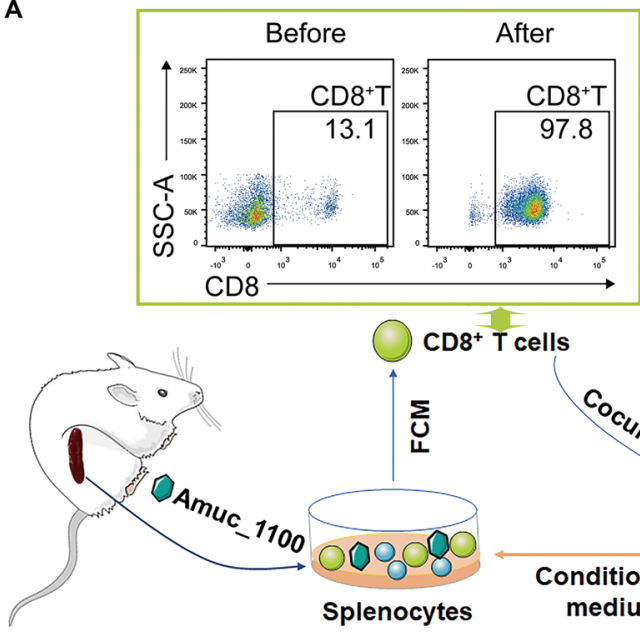

C

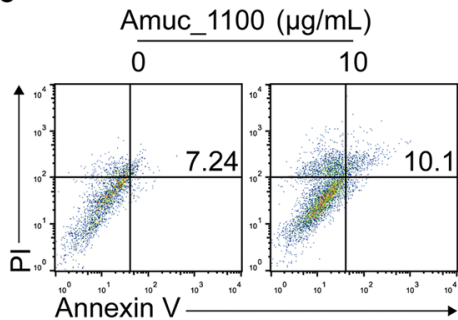

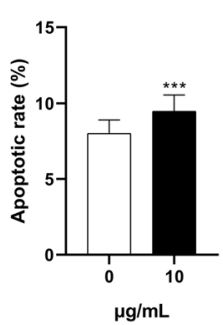

B

Amuc_1100 ( $\mu \mathrm{g} / \mathrm{mL})$

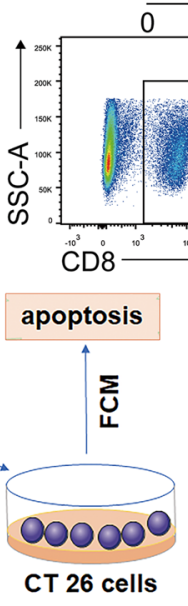

D
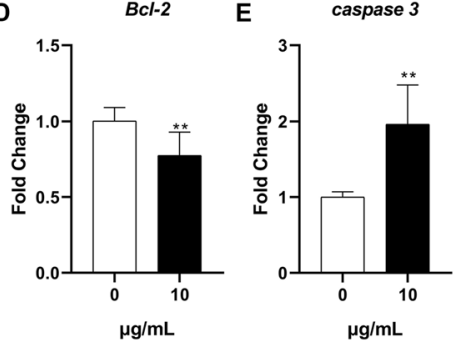

$\mathbf{F}$

AOM+DSS

A.muciniphila+AOM+DSS
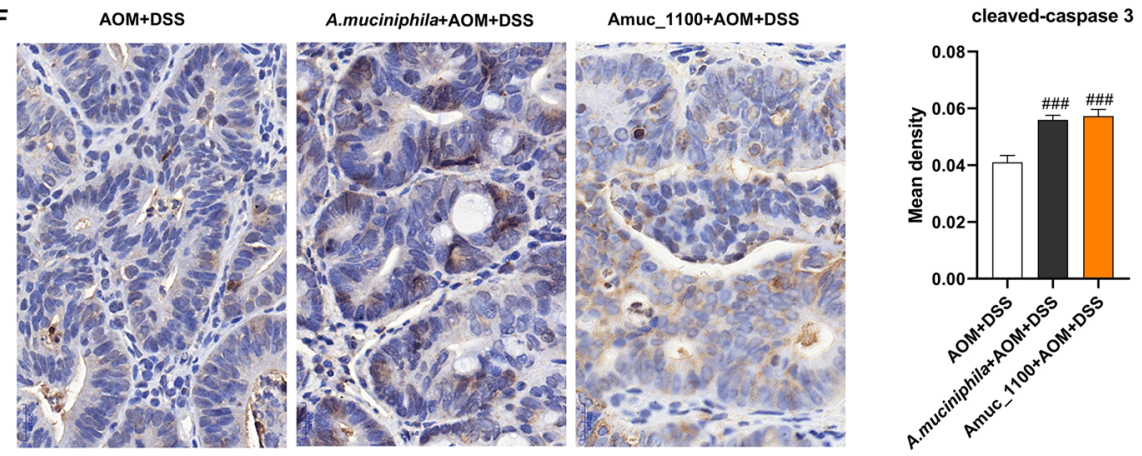

Figure 7 Amuc_1100 induced CTL activation in CT26 cell conditioned medium. (A) Schematic representation of CTL isolation and coculture with CT26 cells. (B) The fraction of CTLs in the splenocytes cultured with CT26 cell conditioned medium with or without Amuc_1100 for 72 hours. (C) The apoptotic rate of CT26 cells cocultured with sorted CTLs for 48 hours $(n=3)$. The mRNA expression of Bcl-2 (D) and caspase 3 (E) and in CT26 cells was detected by qPCR. (F) Intratumoural cleaved-caspase 3 in CAC mice were analysed by IHC. Scale bars, $20 \mu \mathrm{m}$. Data are presented as the means \pm SEM and were analysed with $t$ test. ${ }^{*} \mathrm{P}<0.05,{ }^{*} \mathrm{p}<0.01$, ${ }^{* *} \mathrm{p}<0.001$, compared with control. CAC, colitis-associated colorectal cancer; CTL, cytotoxic T lymphocyte; IHC, immunohistochemistry.

inflammation associated tumourigenesis. Thus, A. muciniphila may be a promising therapeutic target in colitis and CRC.

Acknowledgements The authors would like to thank the participants that have made this research possible. We also thank the Majorbio Bio-pharm Biotechnology Co., Ltd for 165 sequencing and data analysis platform (www.i-sanger.com).

Contributors LW, LT and YF carried out most experiments and analysed the data. $\mathrm{SZ}, \mathrm{MH}$ and $\mathrm{CZ}$ provided clinical sample and performed data analyses. GY and JZ helped with animal experiments and analysis. SC, QW, ZZ and LL designed the experiments and analysed data. ZZ and LL directed the research and wrote the manuscript with input from coauthors.

Funding This project was supported by the National Natural Science Foundations of China (81973096 and 81502801) and Postgraduate Research \& Practice Innovation Program of Jiangsu Province (KYCX18_1513).

\section{Competing interests None declared.}

Patient and public involvement Patients and/or the public were not involved in the design, or conduct, or reporting, or dissemination plans of this research.

Patient consent for publication Not required.
Ethics approval All animal procedures were approved by the Animal Ethical and Welfare Committee of Nanjing Medical University (IACUC-1812031).

Provenance and peer review Not commissioned; externally peer reviewed.

Data availability statement Data are available in a public, open access repository. All data relevant to the study are included in the article or uploaded as supplementary information. The $16 \mathrm{~S}$ sequencing data from human and mice faeces had been submitted to NCBI (Sequence Read Archive, SRA). This BioProject accession number is PRJNA596333 (https://www.ncbi.nlm.nih.gov/bioproject/ PRJNA596333).

Open access This is an open access article distributed in accordance with the Creative Commons Attribution Non Commercial (CC BY-NC 4.0) license, which permits others to distribute, remix, adapt, build upon this work non-commercially, and license their derivative works on different terms, provided the original work is properly cited, appropriate credit is given, any changes made indicated, and the use is non-commercial. See: http://creativecommons.org/licenses/by-nc/4.0/.

\section{ORCID iD}

Zhan Zhang http://orcid.org/0000-0002-9685-5753 


\section{REFERENCES}

1 Huyghe JR, Bien SA, Harrison TA, et al. Discovery of common and rare genetic risk variants for colorectal cancer. Nat Genet 2019;51:76-87.

2 Carr PR, Weigl K, Jansen L, et al. Healthy lifestyle factors associated with lower risk of colorectal cancer irrespective of genetic risk. Gastroenterology 2018;155:1805-15.

3 Grivennikov SI. Inflammation and colorectal cancer: colitis-associated neoplasia. Semin Immunopathol 2013;35:229-44.

4 Zhu W, Miyata N, Winter MG, et al. Editing of the gut microbiota reduces carcinogenesis in mouse models of colitis-associated colorectal cancer. J Exp Med 2019:216:2378-93.

5 Choi C-HR, Al Bakir I, Ding Nik-Sheng (John), et al. Cumulative burden of inflammation predicts colorectal neoplasia risk in ulcerative colitis: a large singlecentre study. Gut 2019;68:414-22

6 Chen J, Pitmon E, Wang K, Microbiome WK. Microbiome, inflammation and colorectal cancer. Semin Immunol 2017;32:43-53.

7 Larabi A, Barnich N, Nguyen HTT. New insights into the interplay between autophagy, gut microbiota and inflammatory responses in IBD. Autophagy 2020;16:1-14.

8 Yang Y, Weng W, Peng J, et al. Fusobacterium nucleatum increases proliferation of colorectal cancer cells and tumor development in mice by activating Toll-like receptor 4 signaling to nuclear Factor $-\kappa \mathrm{B}$, and up-regulating expression of microRNA-21. Gastroenterology 2017;152:851-66.

9 Yu T, Guo F, Yu Y, et al. Fusobacterium nucleatum promotes chemoresistance to colorectal cancer by modulating autophagy. Cell 2017;170:548-63.

10 Long X, Wong CC, Tong L, et al. Peptostreptococcus anaerobius promotes colorectal carcinogenesis and modulates tumour immunity. Nat Microbiol 2019;4:2319-30.

11 Miller RS, Hoskins LC. Mucin degradation in human colon ecosystems. Fecal population densities of mucin-degrading bacteria estimated by a "most probable number" method. Gastroenterology 1981;81:759-65.

12 Derrien M, van Passel MW, van de Bovenkamp JH, et al. Mucin-bacterial interactions in the human oral cavity and digestive tract. Gut Microbes 2010;1:254-68.

13 Everard A, Belzer C, Geurts L, et al. Cross-Talk between Akkermansia muciniphila and intestinal epithelium controls diet-induced obesity. Proc Natl Acad Sci U S A 2013; 110:9066-71.

14 Dao MC, Everard A, Aron-Wisnewsky J, et al. Akkermansia muciniphila and improved metabolic health during a dietary intervention in obesity: relationship with gut microbiome richness and ecology. Gut 2016;65:426-36.

15 Yassour M, Lim MY, Yun HS, et al. Sub-clinical detection of gut microbial biomarkers of obesity and type 2 diabetes. Genome Med 2016;8:17.

16 Depommier C, Everard A, Druart C, et al. Supplementation with Akkermansia muciniphila in overweight and obese human volunteers: a proof-of-concept exploratory study. Nat Med 2019;25:1096-103.

17 Ansaldo E, Slayden LC, Ching KL, et al. Akkermansia muciniphila induces intestinal adaptive immune responses during homeostasis. Science 2019:364:1179-84.

18 Routy B, Le Chatelier E, Derosa L, et al. Gut microbiome influences efficacy of PD-1based immunotherapy against epithelial tumors. Science 2018;359:91-7.

19 Zhang Z, Wu X, Cao S, et al. Chlorogenic acid ameliorates experimental colitis by promoting growth of Akkermansia in mice. Nutrients 2017;9:677.

20 Zhai R, Xue X, Zhang L, et al. Strain-Specific Anti-inflammatory Properties of Two Akkermansia muciniphila Strains on Chronic Colitis in Mice. Front Cell Infect Microbiol 2019;9:239.

21 Plovier H, Everard A, Druart C, et al. A purified membrane protein from Akkermansia muciniphila or the pasteurized bacterium improves metabolism in obese and diabetic mice. Nat Med 2017;23:107-13.

22 XY W, Wang LJ, Tang L, et al. Salvianolic acid B alters the gut microbiota and mitigates colitis severity and associated inflammation. J Funct Foods 2018;46:312-9.

23 Li H, van der Leun AM, Yofe I, et al. Dysfunctional CD8 T cells form a proliferative, dynamically regulated compartment within human melanoma. Cell 2019;176:775-89.

24 Belkaid Y, Harrison OJ. Homeostatic immunity and the microbiota. Immunity 2017:46:562-76.

25 Feng Q, Liang S, Jia H, et al. Gut microbiome development along the colorectal adenoma-carcinoma sequence. Nat Commun 2015;6:6528.
26 Weir TL, Manter DK, Sheflin AM, et al. Stool microbiome and metabolome differences between colorectal cancer patients and healthy adults. PLoS One 2013;8:e70803.

27 Baxter NT, Zackular JP, Chen GY, et al. Structure of the gut microbiome following colonization with human feces determines colonic tumor burden. Microbiome 2014;2:20

28 Kang C-S, Ban M, Choi E-J, et al. Extracellular vesicles derived from gut microbiota, especially Akkermansia muciniphila, protect the progression of dextran sulfate sodium-induced colitis. PLoS One 2013;8:e76520.

29 Zhang T, Li Q, Cheng L, et al. Akkermansia muciniphila is a promising probiotic. Microb Biotechnol 2019:12:1109-25.

30 Ring C, Klopfleisch R, Dahlke K, et al. Akkermansia muciniphila strain ATCC BAA-835 does not promote short-term intestinal inflammation in gnotobiotic interleukin-10deficient mice. Gut Microbes 2019;10:188-203.

31 Lee JC, Lyons PA, McKinney EF, et al. Gene expression profiling of CD8+ T cells predicts prognosis in patients with Crohn disease and ulcerative colitis. J Clin Invest 2011;121:4170-9.

32 Wang Z, Vaughan TY, Zhu W, et al. Gab2 and Gab3 Redundantly Suppress Colitis by Modulating Macrophage and CD8 ${ }^{+}$T-Cell Activation. Front Immunol 2019;10:486.

33 Gronke K, Hernández PP, Zimmermann J, et al. Interleukin-22 protects intestinal stem cells against genotoxic stress. Nature 2019;566:249-53.

34 Fridman WH, Zitvogel L, Sautès-Fridman C, et al. The immune contexture in cancer prognosis and treatment. Nat Rev Clin Oncol 2017;14:717-34.

35 Nosho K, Baba Y, Tanaka N, et al. Tumour-Infiltrating T-cell subsets, molecular changes in colorectal cancer, and prognosis: cohort study and literature review. J Pathol 2010;222:350-66

36 Tanoue T, Morita S, Plichta DR, et al. A defined commensal Consortium elicits CD8 T cells and anti-cancer immunity. Nature 2019;565:600-5.

37 Thomas DA, Massagué J. Tgf-Beta directly targets cytotoxic T cell functions during tumor evasion of immune surveillance. Cancer Cell 2005;8:369-80.

38 Bourboulia D, Stetler-Stevenson WG, metalloproteinases M. Matrix metalloproteinases (MMPs) and tissue inhibitors of metalloproteinases (TIMPs): positive and negative regulators in tumor cell adhesion. Semin Cancer Biol 2010;20:161-8

39 Bhattacharya N, Yuan R, Prestwood TR, et al. Normalizing Microbiota-Induced Retinoic Acid Deficiency Stimulates Protective CD8(+) T Cell-Mediated Immunity in Colorectal Cancer. Immunity 2016;45:641-55

40 Lu J-C, Zeng H-Y, Sun Q-M, et al. Distinct PD-L1/PD1 profiles and clinical implications in intrahepatic cholangiocarcinoma patients with different risk factors. Theranostics 2019:9:4678-87

41 Wang X, He Q, Shen $\mathrm{H}$, et al. TOX promotes the exhaustion of antitumor CD8 ${ }^{+}$ T cells by preventing PD1 degradation in hepatocellular carcinoma. J Hepatol 2019;71:731-41.

42 Matson V, Fessler J, Bao R, et al. The commensal microbiome is associated with antiPD-1 efficacy in metastatic melanoma patients. Science 2018;359:104-8.

43 Yassin M, Sadowska Z, Djurhuus D, et al. Upregulation of PD-1 follows tumour development in the AOM/DSS model of inflammation-induced colorectal cancer in mice. Immunology 2019;158:35-46.

44 Fakih $\mathrm{M}$, Ouyang C, Wang C, et al. Immune overdrive signature in colorectal tumor subset predicts poor clinical outcome. J Clin Invest 2019;129:4464-76.

45 Ngiow SF, Young A, Jacquelot N, et al. A threshold level of Intratumor CD8+ T-cell PD expression dictates therapeutic response to anti-PD1. Cancer Res 2015;75:3800-11.

$46 \mathrm{Na}$ YR, Stakenborg M, Seok SH, et al. Macrophages in intestinal inflammation and resolution: a potential therapeutic target in IBD. Nat Rev Gastroenterol Hepatol 2019;16:531-43

47 Liu P-S, Wang H, Li X, et al. $\alpha$-ketoglutarate orchestrates macrophage activation through metabolic and epigenetic reprogramming. Nat Immunol 2017;18:985-94.

48 Nishimura S, Manabe I, Nagasaki M, et al. Cd8+ effector T cells contribute to macrophage recruitment and adipose tissue inflammation in obesity. Nat Med 2009:15:914-20.

49 Pascual-García M, Bonfill-Teixidor E, Planas-Rigol E, et al. LIF regulates CXCL9 in tumor-associated macrophages and prevents $C D 8^{+} \mathrm{T}$ cell tumor-infiltration impairing anti-PD1 therapy. Nat Commun 2019;10:2416 